\title{
Rational-Choice-Erklärungen zum Rauchverhalten und ihre empirische Relevanz ${ }^{1}$
}

\author{
Von Christiane Gross und Jochen Groß
}

Zusammenfassung: Was leisten Theorien rationaler Wahl hinsichtlich der Erklärung des Rauchverhaltens? Zur Beantwortung dieser Frage werden zwei theoretische Ansätze des RationalChoice-Paradigmas vorgestellt: Gewohnheits- und Suchttheorien und der Humankapitalansatz. Die hieraus abgeleiteten Hypothesen werden mit vier Datensätzen (Eurobarometer 1995, Bundesgesundheits-Survey 1998, telefonischer Gesundheitssurvey 2003 und ALLBUS 2004) getestet. Die hierfür verwendeten Hurdle-Regressionen erlauben nicht nur die qualitative Unterscheidung zwischen Rauchern und Nichtrauchern, sondern behandeln auch die Anzahl der konsumierten Zigaretten als Zählvariable. Es zeigen sich überwiegend stabile Effekte von Alter, Geschlecht und Bildung auf den Rauchstatus (Raucher versus Nichtraucher) und auf die durchschnittlich konsumierte Zigarettenmenge von Rauchern. Dagegen sind Erwerbslose zwar eher Raucher als Erwerbstätige, sie rauchen aber nicht mehr Zigaretten täglich. Die Effekte von Familienstand, Einkommen, und Wohnortgröße variieren stark je nach verwendetem Datensatz, was unterschiedliche Ergebnisse in bisherigen Publikationen zu dem Thema erklären dürfte. Obwohl Suchtverhalten intuitiv als Anomalie des Rational-Choice-Paradigmas erscheint, zeigt die Analyse, dass dieser Ansatz brauchbare Hypothesen zur Erklärung des Rauchverhaltens liefert.

\section{Einleitung}

In Deutschland raucht derzeit mehr als ein Viertel der über 15-Jährigen, wobei knapp 33 Prozent der Männer und 22 Prozent der Frauen rauchen (Mikrozensus 2005²). Die möglichen gesundheitlichen Schäden sind beträchtlich. An den Folgen des Rauchens sterben jährlich mindestens 110.000 Menschen in Deutschland, vor allem in Folge von ursächlich auf das Rauchen zurückzuführenden Krebs- und Herzkreislauferkrankungen. ${ }^{3}$ Dies entspricht in etwa 13 Prozent aller Todesfälle. Im Durchschnitt hat ein langjähriger Raucher eine um acht Jahre kürzere Lebenserwartung als ein Nichtraucher. Damit stellt das Rauchen den größten Einzelgesundheitsrisikofaktor dar und verursacht mehr vorzeitige Todesfälle als AIDS, harte Drogen (z.B. Kokain, Heroin), Alkohol, Autounfälle, Gewaltverbrechen und Selbstmorde zusammengenommen (vgl. Kraus / Augustin 2001; Molarius et al. 2001; Peto et al. 1994: 88ff, 1996).

Diese drastischen Folgen des Rauchens zeigen einerseits die Notwendigkeit gesundheitspolitischer Maßnahmen, verlangen andererseits jedoch nach einer fundierten Erklärung des Rauchverhaltens, erscheint doch der Konsum von Tabakprodukten vor dem Hintergrund dieser Erkenntnisse als vermeintlich irrationales und überraschendes Phänomen. Zwar liegen zahlreiche empirische Befunde zu Einflussgrößen auf das Rauchverhalten vor, allerdings sind bezüglich dieser Untersuchungen drei zentrale Mängel zu konstatieren: Erstens fehlt es an einer soziolo-

1) Unser herzlicher Dank gilt Christian Ganser, Thomas Hinz, Monika Jungbauer-Gans, Peter Kriwy, Thomas Wimmer sowie den anonymen Gutachtern für wertvolle Hinweise zu früheren Versionen des Manuskripts.

2) Angaben des Statistischen Bundesamts, abgerufen am 08.08.2007 unter http://www.destatis.de/jetspeed/portal/cms/Sites/destatis/Internet/DE/Content/Statistiken/Gesundheit/GesundheitszustandRisiken/Tabellen/Content75/Rauchverhalten,templateId=renderPrint.psml.

3) Zur Schätzung der ursächlich auf das Rauchen zurückzuführenden Todesfälle vergleicht man die Inzidenzraten (Anzahl der Neuerkrankungen dividiert durch die Individuenzahl) bestimmter Krankheiten bei Rauchern mit den Inzidenzraten dieser Krankheiten bei Nichtrauchern (vgl. dazu einführend Brenner et al. 2002: 12ff; für entsprechende Befunde John / Hanke 2001). 
gischen Theoriefundierung der vorliegenden Arbeiten, die eine überzeugende Erklärung von Gewohnheits- und Suchtverhalten liefert. Bisher reichen die Arbeiten über die Benennung medizinisch-physiologischer und psychologischer Erklärungsfaktoren (vgl. hierzu bspw. Gardner / David 1999; Gjelsvik 1999) nicht hinaus. Zweitens fokussieren die vorliegenden Studien zumeist entweder den Raucheinstieg oder das Rauchverhalten. Eine qualitative Unterscheidung zwischen Rauchstatus und Rauchverhalten im Rahmen einer Analyse wurde bisher noch nicht vorgelegt. Schließlich sind drittens methodische Mängel nicht nur bei der Durchführung einzelner Studien zu kritisieren, sondern auch bei den herangezogenen Auswertungsverfahren, indem etwa der Zähldatencharakter der konsumierten Zigarettenanzahl vernachlässigt wurde.

Wir versuchen daher in einem ersten Schritt den Rauchstatus sowie das Rauchverhalten theoretisch zu erklären und empirisch prüfbare Hypothesen abzuleiten. Als Ausgangspunkt unserer Überlegungen bieten sich Ansätze an, die dem methodologischen Individualismus folgen, da zum einen Rauchen als Konsum privater Güter aufgefasst werden kann und sich zum anderen aus diesen Ansätze empirisch prüfbare Hypothesen spezifizieren lassen. Diese werden in einem zweiten Schritt mit bedeutenden Datensätzen für Deutschland zu diesem Thema (Eurobarometer 1995; Bundesgesundheits-Survey 1998; telefonischer Gesundheitssurvey 2003 und ALLBUS 2004) empirisch mit angemessenen Verfahren getestet. Durch den Rückgriff auf diese vier Datensätze soll überprüft werden, ob die gefundenen Effekte, die durch unterschiedliche Befragungssituationen und Erhebungsinstrumenten generiert wurden, robust sind.

Zunächst wird knapp der Forschungsstand zu Einflussgrößen auf den Raucheinstieg sowie das Rauchverhalten skizziert (Abschnitt 2). Daran schließen theoretische Ausführungen an, welche sich auf Modelle zur Erklärung von Gewohnheit und Sucht sowie auf die Humankapitaltheorie beziehungsweise Überlegungen zum health capital stützen (Abschnitt 3). In Abschnitt 4 werden die verwendeten Datensätze und die Operationalisierungen der für die Analysen relevanten Variablen vorgestellt sowie einige deskriptive Kennzahlen geliefert. Nachfolgend wird das verwendete Verfahren der Hurdle-Regression vorgestellt (Abschnitt 5), bevor in Abschnitt 6 die Ergebnisse der Schätzungen präsentiert werden. Abschließend werden die Ergebnisse sowohl vor dem theoretischen Hintergrund als auch aus methodischer Sicht diskutiert und Folgerungen für die weitere Forschung gezogen.

\section{Stand der Forschung}

Der Forschungsstand bezüglich des Rauchens ist sicher nicht zuletzt aufgrund der gesellschaftspolitischen Relevanz und der zahlreichen am Phänomen interessierten Disziplinen (Epidemiologie, Pharmakologie, Psychologie, Ökonomik, Soziologie) sehr vielfältig. Im Folgenden kann nur ein Ausschnitt der vorhandenen Forschung behandelt werden. Wir beziehen uns ausschließlich auf die quantitativen Forschungsergebnisse zum Thema Rauchen bei Erwachsenen der letzten 20 Jahre in Deutschland und berücksichtigen lediglich Studien, die soziale Einflussgrößen thematisieren. Trotz der umfangreich vorliegenden Literatur ist auffällig, dass ein systematischer Test von theoretisch abgeleiteten Hypothesen, die wesentliche Einflussfaktoren auf die Rauchentscheidung oder das Rauchverhalten benennen, für Deutschland nicht vorliegt. ${ }^{4}$ Vielmehr findet sich eine Sammlung ad-hoc postulierter und empirisch gefundener Einflussfaktoren, denen zumeist keine theoretische Erklärung zugrunde liegt. ${ }^{5}$

4) Betrachtet man den internationalen Forschungsstand, so zeigt sich, dass insbesondere in den USA auf Grundlage der in Abschnitt 3 behandelten Gewohnheits- und Suchttheorien zahlreiche hypothesenprüfende Studien vorliegen (vgl. etwa Becker 1996; Becker et al. 1991, 1994; Chaloupka 1991).

5) Ausnahmen bestätigen die Regel. So finden sich beispielsweise bei Eifler $(1997,1998)$ Analysen auf Grundlage von sozialpsychologischen Überlegungen zur Selbstkontrolle (Gottfredson / Hirschi 1990) und dem Ansatz der differenziellen Assoziation (Burgess / Akers 1966). Eifler greift dabei auf Daten einer schriftlichen Befragung von 837 Erwachsenen zurück. 
Warschburger (2002) hat den Einfluss relevanter Bezugspersonen auf das Rauchverhalten untersucht. Die Korrelation des dichotom erfassten Rauchstatus von Ehepartnern liegt nach ihren Berechnungen in der Unterstichprobe des Mikrozensus 1995 bei 0,35. Unklar ist jedoch, inwiefern sich der Zusammenhang auf Homophilietendenzen in der Partnersuche zurückführen lässt oder durch Angleichungstendenzen im Zusammenleben entsteht. Nach Kraus et al. (2004) stellt bei Jugendlichen das Rauchverhalten der Freunde eine wesentliche Einflussgröße auf die Rauchprävalenz dar. Auch die Studien der Bundeszentrale für gesundheitliche Aufklärung (2006: 36ff) stellen fest, dass sich jugendliche Raucher häufiger in einem sozialen Umfeld (Haushalt, Freunde) bewegen, das überwiegend raucht.

Des Weiteren korreliert nach Helmert et al. (2001) die Größe der Gemeinde, in der der potenzielle Raucher lebt, mit seinem Rauchstatus. Nach ihren Analysen des Mikrozensus 1995 liegt der Anteil der Raucher in Städten mit über 500.000 Einwohnern deutlich über dem in kleineren Gemeinden. Ebenso finden Völzke et al. (2006) erhebliche regionale Unterschiede in der Rauchprävalenzrate, die über verschiedene betrachtete Datensätze hinweg konsistent sind. Sehr deutlich zeigen sich in den Studien geschlechtsspezifische Unterschiede in der Rauchprävalenz. So liegt der Anteil der Raucher unter Männern deutlich über dem Prozentsatz der rauchenden Frauen (Helmert et al. 2001; Lampert / Burger 2005). Bereits an dieser Stelle soll darauf hingewiesen werden, dass eine theoretisch zufrieden stellende Erklärung der persistenten Geschlechtsunterschiede in der Rauchprävalenz nicht vorliegt, allenfalls einige Hinweise auf mögliche Ursachen (vgl. hierzu etwa Dicken 1978; Elkind 1985). Waldron (1991) identifiziert verschiedene Gründe, die sich auch in einer ersten Analyse als empirisch haltbar erweisen. Ihr zufolge sind es zum einen biologische Unterschiede zwischen Männern und Frauen und zum anderen verschiedene Geschlechterrollen und gesellschaftliche Erwartungen an das Verhalten von Frauen und Männern, die die zu beobachtenden geschlechtsspezifischen Prävalenzen bedingen. Ebenfalls mit den Daten des Mikrozensus 1995 kann gezeigt werden, dass der Anteil der Raucher bei beiden Geschlechtern von 18 bis 30 Jahren ansteigt und ab dem 40. Lebensjahr stetig sinkt (Helmert et al. 2001). Wenngleich der Anteil der Raucher unter Männern deutlich über dem Prozentsatz der rauchenden Frauen liegt (ebd.), nähern sich die geschlechtsspezifischen Raucheranteile in den jüngeren Alterskohorten deutlich an. Auch Lampert und Burger (2005) können diesen Befund auf Grundlage ihrer Auswertungen des telefonischen Gesundheitssurveys 2003 bestätigen. Dies ist sowohl auf den Rückgang der Raucher unter Männern als auch auf den steigenden Anteil der rauchenden Frauen zurückzuführen, wie Kraus und Augustin (2001) anhand ihrer Auswertungen der „Repräsentativerhebung zum Gebrauch psychoaktiver Substanzen bei Erwachsenen in Deutschland" von 2000 feststellen (für denselben Befund vgl. auch Junge / Nagel 1999). Auch das durchschnittliche Einstiegsalter der Frauen liegt dem telefonischen Gesundheitssurvey von 2003 zufolge mit knapp 16 Jahren in den Geburtskohorten 1970 bis 1985 nahe dem der gleichaltrigen Männer (Lampert / Burger 2004). In den jüngeren Geburtskohorten liegt der Anteil der regelmäßigen Raucher bei den 15-jährigen Mädchen schon über dem Anteil der männlichen Mitschüler (Godeau et al. 2004). Ferrence (1988) versucht diese Befunde theoretisch zu erfassen, indem er ein Diffusionsmodell von Innovationen zur Erklärung geschlechtsspezifischer Unterschiede im Rauchverhalten entwickelt. Danach sind Männer innovationsfreudiger als Frauen, womit sich etwa erklären ließe, warum sich die Prävalenzraten der Frauen langsam denen der Männer angleichen. Als Innovation fasst Ferrence bestimmte Verhaltensweisen auf, so etwa das Rauchen am Anfang des 20. Jahrhunderts. Waldron (1991) zeigt jedoch, dass Ferrences Modell empirisch nicht haltbar ist.

Sowohl die Art des Lebensunterhalts beziehungsweise der Erwerbsstatus (Arbeitslosenstatus / Sozialhilfe versus Erwerbstätigkeit) als auch der Berufszweig stehen in Zusammenhang mit dem Rauchverhalten. So ist der Anteil der Raucher unter Arbeitslosen und Sozialhilfeempfängern auf Datenbasis des Mikrozensus 1995 signifikant höher als in der Vergleichs- 
gruppe (Helmert et al. 2001). Nach Helmert (1999) fangen Arbeitslose nicht häufiger an zu rauchen, sondern es fällt ihnen schwerer aufzuhören (vgl. auch Helmert / Maschewsky-Schneider 1998). Helmert und Borgers (1998) beschreiben die berufsspezifischen Unterschiede mit Hilfe der Extremwerte: Der Raucheranteil von 65 Prozent unter Transportarbeitern im Alter von 18 bis 34 Jahren steht hier im Gegensatz zu demjenigen der gleichaltrigen Gymnasiallehrer von acht Prozent. Darüber hinaus machen Helmert et al. (2001) mit Daten des Mikrozensus 1995 einen negativen Effekt des Haushaltsnettoeinkommens auf die Prävalenz des Zigarettenrauchens ausfindig: Je geringer das relative Haushaltsnettoeinkommen (Prozent des Durchschnittseinkommens), desto höher die Konsumprävalenz. Insgesamt kann die Schichtspezifität des Rauchverhaltens als empirisch gut belegt betrachtet werden, wobei die Analysen mit dem telefonischen Gesundheitssurvey 2003 zeigen, dass Männer und Frauen aus der „Unterschicht" (Lampert / Burger 2005: 1232) häufiger anfangen zu rauchen und es ihnen schwerer fällt wieder aufzuhören (vgl. für weitere diesbezügliche Befunde Lampert / Thamm 2004).

Mit Daten des Mikrozensus 1995 können Helmert et al. (2001) zeigen, dass der Anteil von Rauchern unter Geschiedenen signifikant höher ist als in der Vergleichsgruppe. Lampert und Burger (2005: 1236) bemerken hierzu, dass ,,vor allem die relativ hohen Raucheranteile bei allein lebenden Männern im Alter ab 45 Jahren sowie bei jungen Vätern“ auffallen. Junge Mütter hingegen weisen den Autoren zufolge kein besonders auffälliges Rauchverhalten auf, einzig allein erziehende Mütter rauchen auffallend häufig (ebd.).

Zusammenfassend kann festgehalten werden, dass die vorliegenden empirischen Untersuchungen zahlreiche Einflussfaktoren auf das Rauchen und das Rauchverhalten identifizieren konnten. Dennoch bleiben diese hier aufgezählten Erkenntnisse unbefriedigend: Erstens ist nicht geklärt, wie stark die einzelnen Faktoren im Verhältnis zu den anderen die Entscheidung zu rauchen beziehungsweise das Rauchverhalten beeinflussen. Das liegt insbesondere daran, dass in kaum einer der vorliegenden Arbeiten multivariate Auswertungsverfahren angewandt wurden (eine Ausnahme sind etwa Lampert 2005; Lampert / Burger 2005). Zweitens mangelt es der präsentierten Literatur an einer theoretischen Fundierung, die jedoch zur Ableitung relevanter Einflussgrößen unabdingbar ist.

\section{Theoretische Erklärungen des Rauchverhaltens}

Im Folgenden werden zunächst die Begriffe Gewohnheit und Sucht diskutiert, bevor auf theoretische Ansätze auf Basis des methodologischen Individualismus Bezug genommen wird. Da bisher keine befriedigende theoretische Erklärung des Rauchverhaltens vorliegt und die beschriebenen Befunde sich überwiegend allenfalls auf ad-hoc-Hypothesen stützen, kann im Folgenden nicht der Anspruch erhoben werden, eine vollständige Erklärung des Phänomens liefern zu können. ${ }^{6}$ Vielmehr ist dies ein erster Versuch, zu theoretisch gehaltvollen Erklärungen zu gelangen und hierfür erscheint die gewählte theoretische Perspektive viel versprechend, da Gewohnheits- und Suchtverhalten als Konsum von besonderen privaten Gütern, nämlich Sucht- und Gewohnheitsgütern, gesehen werden kann. Bei der Etablierung von Gewohnheit und Sucht handelt es sich um die Aneinanderreihung individueller Konsumentscheidungen. Diese sollten somit auch in den Mittelpunkt der theoretischen Betrachtung gestellt werden (vgl. Herrnstein / Prelec 1992: 336). Zunächst wird eine individuelle Entscheidung getroffen, ob man überhaupt raucht. Wird diese Entscheidung für das Rauchen ge-

6) Andere theoretische Ansatzpunkte als der hier gewählte sind natürlich ebenso denkbar, werden hier jedoch nicht weiter verfolgt. So könnte man etwa das Rauchverhalten als abweichendes Verhalten auffassen und mit entsprechenden Ansätzen versuchen, empirisch prüfbare Hypothesen abzuleiten. Dies erscheint allerdings erst dann sinnvoll, wenn sich eine Nichtrauchernorm stärker als bislang durchgesetzt haben sollte. 
troffen, so ergibt sich danach die Frage, wie viel geraucht wird und ob man gegebenenfalls wieder aufhört.

Im Rahmen des Rational-Choice-Paradigmas wurden einige Vorschläge zur Erklärung von Gewohnheits- und Suchtverhalten erarbeitet, auf die im Folgenden eingegangen wird. Der Rückgriff auf diese Ansätze ist auch deshalb interessant, da Gewohnheits- und Suchtverhalten zunächst als Anomalie rationalen Handelns erscheint. Somit können gerade durch Betrachtung dieses Phänomens die Grenzen der Reichweite Theorien rationalen Handelns untersucht werden. ${ }^{7}$

\subsection{Kennzeichen des Gewohnheits- und Suchtbegriffs}

Für eine theoretische Erfassung des Rauchverhaltens müssen zunächst die Charakteristika von Gewohnheiten und Sucht identifiziert werden, um sie bei der Modellierung berücksichtigen zu können. Es liegen zwar weder für den Gewohnheits- noch für den Suchtbegriff einheitliche Definitionen vor (Elster / Skog 1999; Grossman 1995), jedoch besteht zumindest hinsichtlich wesentlicher Eigenschaften beider Phänomene weitgehend Konsens.

Sucht wird meistens als ein Spezialfall von Gewohnheit betrachtet und als besonders starke Gewohnheit interpretiert (z.B. bei Becker / Murphy 1988). Demnach ist ein Suchtgut immer auch ein Gewohnheitsgut, ein Gewohnheitsgut jedoch nicht zwingend ein Suchtgut (siehe etwa auch Becker 1992). Sucht (und damit auch Gewohnheit) definiert sich über das Vorhandensein folgender Effekte (vgl. Braun 2002: 102ff):

- Gewöhnungseffekt (tolerance): Um im Zeitverlauf einen gleich bleibenden Nutzen zu erzielen, muss immer mehr konsumiert werden, da bei fortschreitendem Konsum der Nutzeneffekt derselben Handlung sinkt.

- Verstärkungseffekt (reinforcement): Ein höherer Konsum in der Gegenwart steigert den zukünftigen Konsum (Selbstverstärkungstendenz).

- Entzugseffekt (withdrawal): Gewohnheitskonsum geht mit Entzugssymptomatiken bei einer plötzlichen Unterbrechung des Konsums einher.

Auf Ansätze auf Grundlage des methodologischen Individualismus, die explizit die Eigenschaften in das theoretische Modell einbeziehen, wird im Folgenden eingegangen.

\subsection{Gewohnheits- und Suchtmodelle}

Grundlegend für Modelle des Gewohnheits- und Suchtverhaltens ist der Ansatz von Becker und Murphy (1988). ${ }^{8}$ Ausgangspunkt ihrer Überlegungen ist die Annahme eines rationalen Akteurs, der vollkommen informiert ist, vorausschauend plant und seinen Nutzen konsistent über die Zeit maximiert. Sie gehen davon aus, dass der Nutzen eines Akteurs vom Konsum zweier Güter abhängt, wovon eines als Gewohnheitsgut charakterisiert wird und das andere ein normales $\mathrm{Gut}^{9}$ darstellt. Die Modellierung der Eigenschaften des Gewohnheitsgutes erfolgt über die Integration eines Gewohnheitskapitalstocks in die Nutzenfunktion eines Akteurs. Dieser Gewohnheitskapitalstock ist als abdiskontierte Summe aller bisherigen Konsumentschei-

7) Für die Diskussion zur Reichweite der Theorien rationalen Handelns vgl. bspw. Boudon (1998), Elster (1993), Frey und Eichenberger (1989) oder Green und Shapiro (1994).

8) Neben diesem dynamischen Modell gibt es weitere Theorien (bspw. Essers Framingmodell 1990, 2001, 2002), die ebenfalls den Anspruch erheben Gewohnheit zu erklären. Einen Überblick sowie Kritik an diesen Ansätzen bietet Berger (2003: 21ff).

9) Normale oder superiore Güter sind nach der mikroökonomischen Haushaltstheorie solche, nach denen ceteris paribus die Nachfrage bei steigendem Einkommen steigt. 
dungen aufzufassen. Die Abdiskontierung ist notwendig, um der Überlegung Rechnung zu tragen, dass ,Handlungen, die lange zurückliegen, nicht denselben gewohnheitsbildenden Effekt haben wie solche, die erst kürzlich ergriffen wurden“ (Berger 2003: 51f). Damit werden die als wesentlich identifizierten Eigenschaften folgendermaßen im Modell umgesetzt:

- Gewöhnungseffekt: Eine Erhöhung des Gewohnheitskapitalstocks senkt den gegenwärtigen Nutzen einer Einheit des Gewohnheitsgutes.

- Verstärkungseffekt: Ein größerer Gewohnheitskapitalstock steigert den gegenwärtigen Verbrauch des Gewohnheitsgutes und ein höherer Konsum in der Gegenwart steigert den zukünftigen Konsum.

- Entzugseffekt: Eine Reduktion des gegenwärtigen Konsums eines Gewohnheitsgutes bei gegebenem Gewohnheitskapitalstock reduziert den gegenwärtigen Nutzen.

Diese im Vergleich zu klassischen nutzenökonomischen Ansätzen durch die Berücksichtigung der bisherigen Konsumgewohnheiten modifizierte Nutzenfunktion maximiert ein Akteur unter Berücksichtigung seiner individuellen Beschränkungen (Budgetrestriktion). Hierunter ist insbesondere das individuelle Einkommen zu fassen. Darüber hinaus wird die Möglichkeit des Einflusses von Lebenszyklusschocks integriert, die den Gewohnheitskapitalstock sprunghaft erhöhen können (Becker / Murphy 1988: 688ff). Demnach ist zu erwarten, dass Geschiedene und Verwitwete eher rauchen als Verheiratete (Hypothese 1a) und wenn sie rauchen, eine größere Menge Zigaretten pro Tag konsumieren (Hypothese $1 b$ ). Ebenso kann aus diesen Überlegungen die Erwartung abgeleitet werden, dass Arbeitslose eher rauchen als Erwerbstätige (Hypothese 2a) und arbeitslose Raucher durchschnittlich mehr Zigaretten täglich rauchen als erwerbstätige Personen (Hypothese 2b).

Aus der für die Maximierung notwendigen Berücksichtigung der Budgetrestriktion ergeben sich weitere Folgerungen hinsichtlich der Auswirkungen von Änderungen des Einkommens und der Preise des Gewohnheitsgutes auf dessen Konsum (vgl. Becker / Murphy 1988: 685ff). Da in keinem verfügbaren Datensatz der tatsächlich bezahlte Zigarettenpreis erhoben wurde, kann im Folgenden nur der Einfluss des Einkommens auf den Zigarettenkonsum betrachtet werden. ${ }^{10}$ Dem skizzierten Modell zufolge ist zu erwarten, dass die konsumierte Menge an Zigaretten sowie die Rauchwahrscheinlichkeit mit steigendem Einkommen zunimmt (Hypothesen 3a und 3b).

Das Modell von Becker und Murphy (1988) beruht auf teilweise heroischen Annahmen, die für eine mathematische Maximierung der Nutzenfunktion unter Berücksichtigung der Budgetrestriktion notwendig sind. Diese Annahmen sind in vielfältiger Weise kritisierbar, jedoch zeigen Weiterentwicklungen des hier präsentierten Grundmodells, dass einige der restriktiven Annahmen gelockert werden können, ohne dass sich die zentralen Folgerungen ändern. ${ }^{11}$ Zudem reichern diese Modifikationen das Grundmodell weiter an und ermöglichen die Ableitung zusätzlicher Hypothesen, beispielsweise über den Verlauf einer Sucht. ${ }^{12}$ Ein Test dieser Implikationen setzt jedoch entsprechende Längsschnittdaten mit detaillierten Informationen etwa zu Raucherkarrieren voraus, die für Deutschland nicht vorliegen. Dennoch lohnt sich unserer Auffassung nach ein Test der spezifizierten Hypothesen, da in Deutschland bisher noch kein Versuch unternommen wurde, wesentliche Einflussfaktoren auf das Rauch-

10) Der tatsächliche Zigarettenpreis ist nicht so homogen wie oftmals angenommen. Sowohl Zigaretten von Billigdiscountern als auch der Zigarettenpreis in angrenzenden Nachbarländern und auf dem Schwarzmarkt sind deutlich geringer als der handelsübliche Preis. Für die USA haben etwa Becker et al. (1991) entsprechende Untersuchungen vorgelegt und finden empirische Belege für die aus dem Modell gefolgerten Hypothesen.

11) Modellerweiterungen haben Dockner und Feichtinger (1993), Orphanides und Zervos (1995, 1998) sowie Braun und Vanini (2003) vorgelegt.

12) Für eine Übersicht über entsprechende Hypothesen vgl. Berger (2003: 45ff). 
verhalten theoretisch zu erfassen und die empirischen Befunde dieser Tests zumindest einen ersten Schluss auf das Potenzial von rationaler Gewohnheits- und Suchtmodelle zulassen.

\subsection{Humankapital und health capital}

Im Folgenden wird Gesundheit als eine Form von Humankapital in Anlehnung an Becker (1993 [1964]) skizziert und das Nachfragemodell für health capital von Grossman (1972) dargestellt. Rauchen wird dabei als schädliches Gesundheitsverhalten aufgefasst. ${ }^{13}$

Becker (1993) führt im Rahmen der Humankapitaltheorie ein breites Spektrum an Aktivitäten auf, die als Investitionen in das Humankapital bezeichnet werden. Neben Bildung und beruflichen Qualifikationen fallen unter diese Aktivitäten auch Investitionen in die Gesundheit im Rahmen von Gesundheitsverhalten (Becker 1993: 60). Unterstellt wird nun, dass ein Akteur vorausschauend plant und entscheidet, ob er in seine Humankapitalausstattung investieren soll oder nicht. Dazu vergleicht er die ihm entstehenden Kosten durch die Investitionen mit den zu erwartenden Renditen aus dem akkumulierten Humankapital. Je größer die Humankapitalausstattung und damit die Rendite in Form des Einkommens, desto größer ist der Anreiz, ein Leben ohne gesundheitliche Beeinträchtigungen zu führen. In Bezug auf das Rauchverhalten ist abzuleiten, dass mit zunehmendem Einkommen einer Person die Rauchwahrscheinlichkeit abnimmt (entgegen Hypothese $3 a$ ) und mit steigendem Einkommen weniger Zigaretten konsumiert werden (entgegen Hypothese $3 b$ ). Eine Verlängerung der Produktionsphase ohne gesundheitliche Beeinträchtigungen, die durch Mortalität und Morbidität bestimmt wird, lohnt sich dabei insbesondere für jüngere Personen (Becker 1993: 85f). Daraus ergeben sich direkt prüfbare Folgerungen hinsichtlich des Zusammenhangs zwischen dem Alter und dem Rauchverhalten: Zum einen ist zu erwarten, dass mit steigendem Alter die Rauchwahrscheinlichkeit zunimmt (Hypothese 4a) und zum anderen, dass auch die konsumierte Zigarettenmenge positiv mit dem Alter variiert (Hypothese 4b).

Diesen Aspekt betont auch Grossman (1972) im Zuge seines Nachfragemodells nach health capital. Diese Dimension des Humankapitals bestimmt weniger die Produktivität der Arbeit als vielmehr die besagte Zeitspanne, in der Erwerbsarbeit oder jegliche andere Form von Produktion möglich ist. Gesundheit wird dabei sowohl als Konsumgut als auch als Investitionsgut aufgefasst. Grossman (1972) geht von einem Anfangsbestand an Gesundheit aus, der sich mit steigendem Alter mit zunehmender Rate entwertet. Die Kosten für Gesundheitsinvestitionen sinken mit zunehmender Bildung, da höher gebildete Personen aufgrund von Wissen um gesundheitsförderliches und -schädliches Verhalten Gesundheit effizienter produzieren können. Demnach ist zu erwarten, dass mit steigender Bildung die Rauchwahrscheinlichkeit abnimmt (Hypothese 5a). Gleichwohl kann für Raucher postuliert werden, dass mit steigender Bildung die konsumierte Zigarettenmenge abnimmt (Hypothese $5 b)$. Die abgeleiteten Hypothesen werden in Tabelle 1 zusammengefasst. ${ }^{14}$

13) Eine Zusammenfassung mikrosoziologischer Ansätze des Gesundheitsverhaltens findet sich bei Jungbauer-Gans und Gross (2006).

14) Getestet werden im Folgenden demnach die Konsequenzen aus den theoretischen Überlegungen und nicht deren Grundannahmen. Innerhalb der Rational-Choice-Theorie nehmen wir damit die Position ein, die von konstanten individuellen Präferenzen ausgeht, da erstens eine valide Erfassung von Präferenzen mit erheblichen methodischen Problemen verbunden ist und zweitens diese Variablen in den aufgeführten Datensätzen nicht erhoben wurden. Insbesondere Vertreter des SEU-Ansatzes verfolgen im Gegensatz dazu die Strategie, direkt den individuellen Nutzen zu erfassen und versuchen somit einen Test der Grundannahmen der Theorien rationaler Wahl. Einen knappen Überblick über diese unterschiedlichen Positionen liefert Best (2007). Das gleiche methodische Problem gilt im Übrigen auch für die Übertragung des SEU-Ansatzes auf gesundheitsrelevantes Verhalten in Form des Health-Belief-Ansatzes (siehe den von Becker 1974 herausgegebenen Sammelband) und dessen Erweiterungen, deren große Schwäche ebenfalls in der Operationalisierung ihrer Konstrukte - insbesondere Präferenzen, Einstellungen und subjektive Nutzenerwartungen - liegt (zusammenfassend Janz et al. 2002). 
Tabelle 1: Hypothesenüberblick

\begin{tabular}{ll}
\hline Theorie & $\begin{array}{l}\text { Eine hohe Rauchwahrscheinlichkeit und eine große Konsummenge ge- } \\
\text { hen einher mit ... }\end{array}$ \\
\hline $\begin{array}{l}\text { Gewohnheits- und } \\
\text { Suchtmodelle }\end{array}$ & $\begin{array}{l}\text { Hypothese } 1 \ldots \text { dem Familienstand geschieden oder verwitwet } \\
\text { Hypothese } 2 \ldots \text { fehlender Erwerbsarbeit } \\
\text { Hypothese } 3 \ldots \text { steigendem Einkommen }\end{array}$ \\
\hline $\begin{array}{l}\text { Humankapital und } \\
\text { health capital }\end{array}$ & $\begin{array}{l}\text { Hypothese } 3 \ldots \text { sinkendem Einkommen } \\
\text { Hypothese } 4 \ldots \text { steigendem Alter }\end{array}$ \\
& Hypothese $5 \ldots$ geringer Bildung \\
\hline
\end{tabular}

\section{Beschreibung der Datenquellen}

Bevor die theoretisch abgeleiteten Hypothesen getestet werden, wird die dem Test zugrunde liegende Datenbasis knapp vorgestellt. Entsprechend unserer Zielsetzung zu prüfen, ob die spezifizierten Einflussfaktoren auf den Rauchstatus sowie das Rauchverhalten robust gegenüber verschiedenen Erhebungsverfahren sind, greifen wir auf Datensätze zurück, die Angaben zum Rauchstatus und Rauchverhalten umfassen. Im Einzelnen sind dies der Eurobarometer 1995, der Bundesgesundheits-Survey 1998, der telefonische Gesundheitssurvey 2003 sowie die Allgemeine Bevölkerungsumfrage der Sozialwissenschaften 2004.

\subsection{Erhebungsdesigns}

Das Standard-Eurobarometer wird seit 1973 im Auftrag der Generaldirektion für Presse und Kommunikation der Europäischen Kommission zweimal jährlich durchgeführt. Die Erhebung auf nationaler Ebene wird an lokal ansässige Institute weitergegeben, mit der Angabe, einen möglichst repräsentativen Querschnitt der Bevölkerung abzubilden. Pro Teilnehmerland sollen in etwa 1.000 Personen mündlich befragt werden, wobei es in Deutschland im Jahr 1995 jeweils ca. 1.000 Personen in Ost- und in Westdeutschland waren (im Folgenden EB95). Der Schwerpunkt in diesem Jahr lag unter anderem in den Themen Rauchgewohnheiten und Krebsrisiko (European Commission 1995).

Mit dem Bundesgesundheits-Survey aus dem Jahr 1998 (BGS98) führte das Robert-KochInstitut (RKI) den ersten gesamtdeutschen Gesundheitssurvey durch. Die Stichprobenziehung erfolgte geschichtet nach Bundesland und Klassifizierung der Gemeindegröße (BIK). Hierbei wurden Sample Points mit einer Wahrscheinlichkeit proportional zur Einwohnerzahl und disproportional zu Gunsten der neuen Bundesländer gezogen (Bellach et al. 1998; Thefeld et al.1999). ${ }^{15}$ Die Ausschöpfungsquote beträgt insgesamt 61 Prozent und variiert je nach Alters-Geschlechts-Konstellation. Der Kernsurvey beinhaltet einen von dem Probanden selbst auszufüllenden Fragebogen, ein via CAPI durchgeführtes Arztinterview sowie eine medizinische Untersuchung (Thefeld et al. 1999). Dabei werden neben sechs Körpermessgrößen (Gewicht, Größe, etc.) auch zahlreiche Laborwerte bestimmt (Thierfelder et al. 1998). Gerade in Bezug auf manch körperbezogene Werte wie beispielsweise Gewicht unterliegen die so gewonnenen Daten sicherlich weniger Verzerrungen als die von Probanden selbst berichteten Werte anderer Datenquellen. Zudem wird die Messqualität des Kernsurveys durch externe Qualitätskontrollen gesichert (detaillierter bei Winkler et al. 1999).

Der erste telefonische Gesundheitssurvey des Robert-Koch-Instituts aus dem Jahr 2003 (GSTel03) behandelt ähnliche Themenfelder wie der BGS98, versteht sich jedoch als flexi-

15) Näheres zur Feldarbeit ist dem Aufsatz von Potthoff et al. (1999) zu entnehmen. 
ble, schnell zu realisierende und kostengünstige Variante der nationalen Gesundheitsberichterstattung (Kohler / Ziese 2004; Ziese 2005). Mit Hilfe der Gabler-Häder-Methode ${ }^{16}$ wird eine Grundgesamtheit an Festnetztelefonnummern erstellt. Aus dieser Grundgesamtheit wird ein ADM-Mastersample gezogen, das die Haushalte bestimmt. Auf Personenebene wird die Zufallsauswahl durch die next-birthday-Methode sichergestellt (Kohler 2005). Auf diese Weise wurden 8.318 Personen der volljährigen Wohnbevölkerung Deutschlands befragt (Ziese 2005).

Die Allgemeine Bevölkerungsumfrage der Sozialwissenschaften (ALLBUS) wird vom Zentrum für Umfragen, Methoden und Analysen e.V. (ZUMA, Mannheim) und dem Zentralarchiv für Empirische Sozialforschung (ZA, Köln) in Kooperation mit dem ALLBUS-Ausschuss realisiert. ${ }^{17}$ Der Schwerpunkt der Erhebung 2004 (kurz ALLBUS04) liegt unter anderem beim Thema Gesundheit. Die Stichprobenziehung erfolgte zweistufig. Zuerst wurden Gemeinden zufällig ausgewählt. Im Anschluss wurde aus ihren Einwohnermelderegistern eine Personenzufallsstichprobe gezogen. Die Schichtung Ost-West berücksichtigt die neuen Bundesländer überproportional. Mit diesem Vorgehen wurde eine Ausschöpfungsquote von insgesamt 45,7 Prozent erreicht (Ost: 47,6\%, West 44,9\%). ${ }^{18}$ Die Befragung erfolgte im Rahmen eines persönlichen, computerunterstützten Interviews (CAPI) (für den ausführlichen Methodenbericht siehe Haarmann et al. 2006). Tabelle 2 liefert einen Überblick über die im Weiteren verwendeten Datensätze.

Tabelle 2: Überblick über die Datensätze

\begin{tabular}{|c|c|c|c|c|}
\hline & EB95 & BGS98 & GSTel03 & ALLBUS04 \\
\hline Erhebungszeitraum & $\begin{array}{l}\text { März bis } \\
\text { April } 1995\end{array}$ & $\begin{array}{c}\text { Okt. } 1997 \text { bis } \\
\text { März } 1999\end{array}$ & $\begin{array}{l}\text { Sept. } 2002 \text { bis } \\
\text { März } 2003\end{array}$ & $\begin{array}{l}\text { März bis Juli } \\
2004\end{array}$ \\
\hline Befragungsmethode & $\begin{array}{c}\text { mündlich } \\
\text { (face-to-face) }\end{array}$ & $\begin{array}{l}\text { schriftlich, } \\
\text { Ärzteinterview } \\
\text { (CAPI), medizi- } \\
\text { nische Unter- } \\
\text { suchung }\end{array}$ & $\begin{array}{l}\text { telefonisch } \\
\text { (CATI) }\end{array}$ & $\begin{array}{l}\text { mündlich } \\
\text { (CAPI) }\end{array}$ \\
\hline $\begin{array}{l}\text { Altersbegrenzung der } \\
\text { Befragten }\end{array}$ & $\geq 15$ & $18-79$ & $\geq 18$ & $\geq 18$ \\
\hline Ausschöpfungsquote & keine Angabe & $61 \%$ & $59 \%$ & $46 \%$ \\
\hline Realisierte Fallzahl & $2.107^{\mathrm{a}}$ & 7.124 & 8.313 & 2.946 \\
\hline
\end{tabular}

a Es handelt sich dabei um die 2.107 Probanden, die in Deutschland befragt wurden. Die Fallzahl des gesamten Eurobarometers beträgt 16.238.

Anmerkung: Die Quellen der Angaben sind dem Text zu entnehmen.

16) Die Gabler-Häder-Methode zur Generierung einer hypothetischen Grundgesamtheit an Festnetzanschlüssen sieht im ersten Schritt die Bereinigung der registrierten Telefonnummern durch Löschung von Servicenummern, Firmennummern, Telefax-Nummern und doppelten Einträgen vor. Im zweiten Schritt werden die letzten beiden Ziffern der bereinigten Nummernliste gelöscht und durch die Nummernfolge -00 bis -99 ersetzt. Auf diese Weise werden auch die nicht registrierten Telefonanschlüsse berücksichtigt (Gabler / Häder 1998).

17) Das ALLBUS-Programm ist 1980-1986, 1991 von der Deutschen Forschungsgemeinschaft (DFG) gefördert worden. Die weiteren Erhebungen wurden von Bund und Ländern über die Gesellschaft Sozialwissenschaftlicher Infrastruktureinrichtungen (GESIS) finanziert. Die Daten sind beim Zentralarchiv für empirische Sozialforschung (Köln) erhältlich. Die vorgenannten Institutionen und Personen tragen keine Verantwortung für die Verwendung der Daten in diesem Beitrag.

18) Freundlicherweise teilte uns Frau Martina Wasmer (ZUMA) diese Information auf Anfrage am 02.03.2006 via E-Mail mit. 


\subsection{Operationalisierung des Rauchverhaltens}

Für diese Untersuchung ist entscheidend, wie (a) der Rauchstatus und (b) die Anzahl der täglich konsumierten Zigaretten eines Rauchers erhoben wurden.

$\mathrm{Zu}$ (a): In der Regel wird zwischen Nichtrauchern (Nie- und Ex-Rauchern) und Rauchern (regelmäßigen Rauchern und Gelegenheitsrauchern) unterschieden.

Tabelle 3: Operationalisierung Rauchstatus

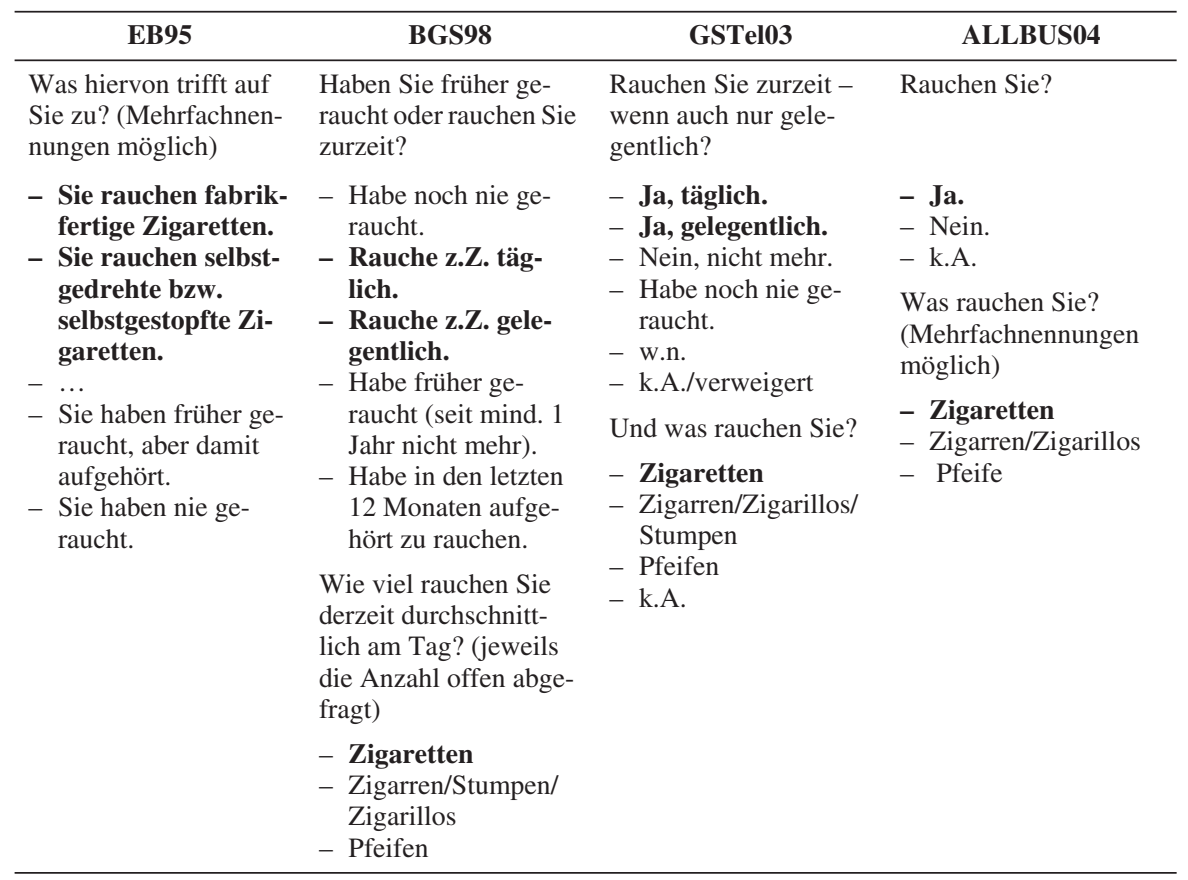

Als Raucher werden im Folgenden diejenigen Personen definiert, die angaben, gelegentlich oder täglich Zigaretten zu rauchen (die detaillierte Erfassung ist den fett markierten Antwortkategorien in Tabelle 3 zu entnehmen). Wir beschränken uns im Rahmen dieses Aufsatzes auf den Konsum von Zigaretten (sowohl fabrikfertige als auch selbst gedrehte und gestopfte). Pfeifen- oder Zigarrenraucher werden nicht berïcksichtigt, da diese nur einen geringen Anteil von fünf Prozent an allen Rauchern ausmachen (Lampert / Burger 2004). Eine gewisse Besonderheit muss hinsichtlich der Abgrenzung von verschiedenen Rauchgütern beim BGS98 beachtet werden. Hier wurde zunächst der Rauchstatus abgefragt (siehe Tabelle 3), danach die durchschnittliche Anzahl täglich gerauchter Zigaretten, Zigarren / Stumpen / Zigarillos und / oder Pfeifen. Nicht berücksichtigt wurden diejenigen die angaben, durchschnittlich keine Zigaretten am Tag zu rauchen, dafür aber von Null abweichende Angaben bei dem Konsum von Zigarren / Stumpen / Zigarillos und / oder Pfeifen machten.

$\mathrm{Zu}$ (b): Die Menge der täglich gerauchten Zigaretten wird in der Regel metrisch erhoben (BGS98, GSTel03, ALLBUS04) im EB95 jedoch nur kategorial erfasst. Die Kategorien des EB95 sind abgestuft mit sieben Intervallen, die jeweils die „Breite“ von fünf Zigaretten aufweisen (5-9, 10-14 ... 35-39) und den Randkategorien „,weniger als 5 Zigaretten“ und „40 Zigaretten und mehr". Im Falle der kategorial erhobenen Zigaretten werden bei den Berech- 
nungen die Kategoriemittelwerte verwendet. Bei den nach oben offenen Kategorien wird jeweils der Durchschnittswert derjenigen Raucher im BGS98 genommen, die 40 Zigaretten und mehr rauchen. Bei den folgenden Analysen wird diese rekodierte Variable als metrisch skaliert betrachtet. ${ }^{19}$

Für die folgenden Analysen mit dem Hurdle-Regressionsmodell wurde aus dem Rauchstatus und der Anzahl durchschnittlich konsumierter Zigaretten am Tag eine einzige Variable gebildet, deren Wert Null die Nichtraucher (Nie- und Ex-Raucher) gemäß der obigen Operationalisierung umfasst. Werte größer Null geben entsprechend die Anzahl der durchschnittlich konsumierten Zigaretten wieder. Zu beachten ist hierbei jedoch, dass die Möglichkeit besteht, sich selbst zunächst als Raucher einzustufen, bei den folgenden Fragen nach der gerauchten Zigarettenanzahl jedoch weniger als eine Zigarette am Tag anzugeben. Bei der Zusammenfassung des Rauchstatus und der konsumierten Zigarettenanzahl wurden diese Nennungen als fehlender Wert betrachtet, da durch die Angaben nicht eindeutig zu entscheiden ist, ob die betroffenen Personen die „Hürde“ zwischen Nichtrauchern und Rauchern überschritten haben oder nicht. Anzumerken ist jedoch auch, dass diese Vorgehensweise keine wesentlichen Auswirkungen auf die Schätzergebnisse hat, da nur ein sehr geringer Anteil der Raucher angab, weniger als eine Zigarette am Tag zu rauchen (in allen Datensätzen betrifft dies - zumeist deutlich - weniger als fünf Prozent der Raucher).

\subsection{Deskriptive Statistik}

Der folgende Überblick in Tabelle 4 gibt die Anteilswerte der Dummy-Variablen wie auch die Durchschnittswerte der metrischen Variablen datensatzspezifisch wieder. Es handelt sich hierbei um die tatsächlichen Kenngrößen der gezogenen Stichproben und nicht um geschätzte Populationsparameter, wie man sie etwa durch Gewichtung erzielen würde. Dieses Vorgehen wird gewählt, da es uns in erster Linie um das Testen von Hypothesen und weniger das Bestimmen von Anteilswerten in der Population geht.

19) Im selben Jahr wie der Eurobarometer wurde eine Zusatzbefragung zum Mikrozensus durchgeführt, die ebenfalls Angaben zum Rauchverhalten beinhaltete. Hier wurde jedoch die tägliche Konsummenge in nur vier verschiedenen Kategorien erhoben. Da diese Variable keinesfalls als metrisch zu betrachten ist und somit ein anderes Schätzmodell verwendet werden müsste als für die anderen Datensätze, wird aufgrund des hier angestrebten Vergleichs der Koeffizienten auf die Analyse des Mikrozensus 1995 verzichtet. Für Analysen mit diesem Datensatz im Hinblick auf das Rauchverhalten vgl. die in Abschnitt 2 erwähnten Studien. 
Tabelle 4: Überblick über die verwendeten Variablen

\begin{tabular}{|c|c|c|c|c|c|}
\hline & Variablen & EB95 & BGS98 & GSTel03 & ALLBUS04 \\
\hline \multirow{10}{*}{ 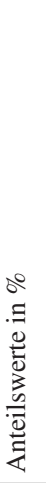 } & Raucher & 28,4 & 32,8 & 32,4 & 28,4 \\
\hline & verheiratet & 61,1 & 66,2 & 58,2 & 61,6 \\
\hline & Geschieden / verwitwet & 18,4 & 12,1 & 16,2 & 14,5 \\
\hline & derzeit arbeitslos & 9,5 & 8,5 & 4,3 & 7,2 \\
\hline & Mittlere Reife & 49,7 & 34,2 & 33,3 & 34,0 \\
\hline & Abitur & 24,6 & 23,3 & 33,5 & 22,9 \\
\hline & Mann & 48,5 & 48,4 & 46,6 & 49,4 \\
\hline & Kind unter $15 \mathrm{im} \mathrm{HH}$ & 27,6 & 34,5 & 19,9 & 26,5 \\
\hline & Stadt $(20.000-100.000)$ & 15,8 & 25,5 & 20,2 & 21,6 \\
\hline & Metropole (> 100.000) & 54,7 & 29,7 & 25,0 & 27,2 \\
\hline \multirow{3}{*}{ 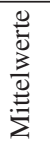 } & Anzahl täglicher Zigaretten bei Rauchern & 16,2 & 15,3 & 14,1 & 15,3 \\
\hline & Alter in Jahren & 39,1 & 46,0 & 46,7 & 47,7 \\
\hline & HH-Einkommen & 1.663 & 2.023 & 2.521 & 2.207 \\
\hline
\end{tabular}

Hinsichtlich der in Tabelle 4 dargestellten Variablen sind einige Besonderheiten und Unterschiede der Erhebung in den jeweiligen Datensätzen zu berücksichtigen. Zunächst betrifft dies die Messung der Schulbildung. Diese wird in den Datensätzen normalerweise über den erzielten Schulabschluss erfasst. Im Eurobarometer jedoch werden aus Gründen internationaler Vergleichbarkeit nicht Schulabschlüsse, sondern nach dem Alter des Probanden gefragt, mit dem er die Schulbildung abgeschlossen hat. Die Kategorien sind dabei „15 Jahre und jünger" für die Referenzkategorie der niedrigen Bildung, ,16 bis 19 Jahre“ für die mittlere Bildung und „20 Jahre und älter“ für die Kategorie hohe Bildung. Die Kategorie „,besucht noch Schule" wurde je nach Alter in die entsprechende Bildungskategorie eingeteilt. Dies erklärt auch den hohen Anteil vermeintlicher Probanden ,,mit mittlerer Reife“.

Die Einkommenserhebung unterscheidet sich nach Datensatz. Das Einkommen im Eurobarometer entspricht dem Bruttohaushaltseinkommen, welches kategorial abgefragt wurde. Ebenfalls kategorial wurde das Einkommen im BGS98 erfasst - hier jedoch das Haushaltsnettoeinkommen. In beiden Fällen wurden für die Bildung des Durchschnittswertes die Kategorienmittelwerte verwendet. Für den BGS98 wurde die nach oben offene größte Einkommenskategorie mit Hilfe der offenen Einkommensabfrage des Datensatzes ALLBUS von 1998 geschätzt. Die Durchschnittswerte der Einkommensmessung sind deswegen und aufgrund fehlender Gewichtung kaum über die Datensätze hinweg zu vergleichen.

Die Schwankungen des Anteils der Arbeitslosen in den unterschiedlichen Datensätzen sind sicherlich nicht als Trendanalyse zu verstehen. Vielmehr ist der auffallend geringe Anteil an arbeitslosen Probanden im GSTel03 bedingt durch die Art der Stichprobenziehung. Bei der Stichprobenziehung des GSTel03 wurde auf eine zu Gunsten der ostdeutschen Wohnbevölkerung disproportionale Auswahlwahrscheinlichkeit verzichtet.

Über die Ursachen des überraschend großen Anteils an Großstädtern im Eurobarometer von 1995 von über 54 Prozent lässt sich nur spekulieren. Denkbar wäre eine verzerrte Teilnahmebereitschaft dahingehend, dass Großstädter an europäischen, internationalen Themen eher interessiert sind als Kleinstädter und Dorfbewohner. 


\section{Das Hurdle-Regressionsmodell}

Bislang wurden zur Schätzung von Zählvariablen - in unserem Fall die Anzahl der Zigaretten - häufig OLS-Regressionen verwendet. Auch wenn lineare Regressionsmodelle häufig zu plausiblen Ergebnissen führen, kann ihre Anwendung bei Zählvariablen zu ineffizienten, inkonsistenten und verzerrten Schätzern führen (Long 1997: 217; Long / Freese 2006: 349). Zudem entspricht die Anwendung von OLS-Regressionen nicht dem Wertebereich der durchschnittlich konsumierten Zigarettenanzahl, da bei OLS-Modellen ein unendlicher Wertebereich unterstellt wird, während hier nur positive, ganzzahlige Werte auftreten können. Davon abgesehen kann die Entscheidungen zu rauchen und darüber, wie viel man durchschnittlich raucht, nicht als unabhängig voneinander betrachten werden, weshalb im Folgenden zur Analyse des Rauchverhaltens ein Hurdle-Modell verwendet wird.

Es handelt sich dabei um ein zweistufiges Zählvariablen-Modell. Im ersten Schritt wird die Wahrscheinlichkeit dafür geschätzt, dass die abhängige Zählvariable (Anzahl der durchschnittlich am Tag konsumierten Zigaretten) einen Wert größer Null annimmt. In unserem Fall liegt die Hürde zwischen Null und einer Zigarette. Das binäre Teilmodell unterscheidet zwischen $\mathrm{y}=0$ und $\mathrm{y}>0$ und kann beispielsweise mit einem Logit-Modell geschätzt werden (Long / Freese 2006: 387). Im zweiten Schritt wird die Ausprägung der Zählvariablen als bedingte Wahrscheinlichkeit mit einer um Null gestutzten Poissonverteilung oder einer um Null gestutzten negativen Binomialverteilung geschätzt (Cameron / Trivedi 1998: 123; Long / Freese 2006: 387). ${ }^{20} \mathrm{Da}$ in jedem der vier Datensätze die Varianz deutlich größer als der Mittelwert ist (Overdispersion), ${ }^{21}$ verwenden wir die negative Binomialregression zur Schätzung der Parameter, da bei Overdispersion die Poissonregression nach unten verzerrte Standardfehler schätzen würde (Long / Freese 2006: 376).

\section{Ergebnisse}

Im Folgenden werden die Ergebnisse zu den beiden Fragestellungen präsentiert, welche Variablen die Chance, dass eine Person gegenwärtig raucht, beeinflussen und welche Variablen einen Effekt auf die durchschnittliche Menge der täglichen Zigaretten eines Rauchers haben. Die unabhängigen Variablen sind jeweils identisch für die beiden Schritte der Hurdle-Regression (vgl. Abschnitt 3). Diese theoretisch hergeleiteten Einflussgrößen werden durch die Kontrollvariablen Geschlecht, Wohnortgröße und Anwesenheit einer Person unter 15 Jahren im Haushalt ergänzt.

Wie die Ausführungen zum Forschungsstand gezeigt haben, wurde der umgekehrt u-förmige Zusammenhang zwischen der Anzahl der Zigaretten und dem Lebensalter bereits belegt. Entgegen der Hypothesen zu Humankapital und health capital, die einen linearen Zusammenhang postulieren (Hypothesen $4 \mathrm{a}$ und $4 \mathrm{~b}$ ), rechnen wir die Modelle ebenfalls mit einem zusätzlichen quadrierten Altersterm. Für den Rauchstatus wurde mit allen vier Datensätzen jeweils ein Likelihood-Ratio-Test durchgeführt, der ein logistisches Regressionsmodell mit einem zusätzlichen quadrierten Altersterm mit einem logistischen Regressionsmo-

20) Statistisch gesehen erscheint diese Modellierung insbesondere notwendig, da man empirisch häufig den Fall vorfindet, dass der Wert Null zu häufig auftritt, um adäquat eine Poisson- oder auch negativ Binomialregression zu schätzen (Winkelmann 2003: 138). Eine Alternative zu dem verwendeten Hurdle-Verfahren stellen spezielle Zähldatenverfahren für zero inflation dar (Cameron / Trivedi 1998: 123ff; Long / Freese 2006: 394ff; Winkelmann 2006: 148ff; für eine empirische Anwendung für den Zigarettenkonsum vgl. Bauer et al. 2006). Aufgrund theoretischer Überlegungen, wonach es sich in unserem Fall um einen zweistufigen Entscheidungsprozess handelt, wurde für die Analysen dem Hurdle-Modell der Vorzug gegeben.

21) Für alle Datensätze wurde ein Likelihood-Ratio-Test zur Überprüfung, ob Overdisperison vorliegt, durchgeführt. In allen Fällen war dieser Test höchst signifikant. 
dell ohne diesen zusätzlichen Term vergleicht. Konsistent zeigt sich, dass die Modelle mit dem quadrierten Altersterm höchst signifikant besser sind. Auch für das Zähldatenmodell zeigt sich konsistent über alle herangezogenen Datensätze hinweg, dass die Aufnahme des quadrierten Altersterms zu einer Verbesserung der Schätzung führt.

Tabelle 5: Einflüsse auf den Rauchstatus und die Anzahl der konsumierten Zigaretten

\begin{tabular}{|c|c|c|c|c|c|}
\hline & EB95 & BGS98 & GSTel03 & $\begin{array}{c}\text { ALLBUS } \\
04\end{array}$ & $\begin{array}{l}\text { Hypot- } \\
\text { hesen }\end{array}$ \\
\hline \multicolumn{6}{|l|}{ Hurdle } \\
\hline geschieden / verwitwet ${ }^{\text {(a) }}$ &, 383 &, $354 *$ &, $337 * *$ &, 353 & H1a: + \\
\hline verheiratet $^{(a)}$ &,- 044 &,- 188 &,$- 404 * * *$ &,$- 482 * *$ & \\
\hline derzeit arbeitslos & ,376* &, $228^{*}$ &, $481 * * *$ &, $523 * *$ & H2a: + \\
\hline HH-Einkommen in $1000 €$ &,- 023 &,$- 104 * * *$ &,- 036 &,- 074 & H3a: +/- \\
\hline Alter &, 044 &, $034^{*}$ &, $065^{* * *}$ &, $087^{* * * *}$ & H4a: + \\
\hline quadriertes Alter &,$- 001 * *$ &,$- 001 * * *$ &,$- 001 * * *$ &,$- 001 * * *$ & H4a: n.s. \\
\hline Mittlere Reife $^{(b)}$ &, 026 &,$- 174 *$ &,$- 223 * *$ &,$- 408 * *$ & H5a: - \\
\hline Abitur (b) &,$- 696^{* * *}$ &,$- 675^{* * *}$ &,$- 814 * * *$ & $-1,204 * * *$ & H5a: - \\
\hline Geschlecht (1 = männlich) &, $977 * * *$ &, $356^{* * *}$ &, $157 * *$ &, $545^{* * * *}$ & \\
\hline Kind unter $15 \mathrm{im} \mathrm{HH}$ & ,205 &, 025 &,- 018 & ,029 & \\
\hline Kleinstadt ${ }^{(c)}$ &, 071 &, $183^{*}$ &, 030 &, 165 & \\
\hline Großstadt ${ }^{(d)}$ &, 068 &, $233 * *$ &, $151^{*}$ &, $249^{*}$ & \\
\hline Konstante & $-1,448 * *$ &,- 170 &,$- 635^{*}$ & $-1,287 * *$ & \\
\hline \multicolumn{6}{|l|}{ Frequency } \\
\hline geschieden / verwitwet ${ }^{\text {(a) }}$ &,- 028 & ,092 & ,014 &,- 008 & H1b: + \\
\hline verheiratet ${ }^{(a)}$ &,- 136 &,- 049 &,$- 087 *$ &,- 061 & \\
\hline derzeit arbeitslos &,- 032 &, 051 &, 057 &, 061 & $\mathrm{H} 2 \mathrm{~b}:+$ \\
\hline HH-Einkommen in $1000 €$ &, 053 &,- 005 & 014 &,- 001 & H3b: +/- \\
\hline Alter &, $040 * * *$ &, $057 * * *$ &, $048 * * *$ &, $046^{* * * *}$ & H4b: + \\
\hline quadriertes Alter &,$- 000 * * *$ &,$- 001 * * *$ &,$- 001 * * *$ &,$- 000 * * *$ & H4b: n.s. \\
\hline Mittlere Reife ${ }^{(b)}$ &,- 015 &,$- 218 * * *$ &,$- 155^{* * *}$ &,- 077 & H5b: - \\
\hline Abitur ${ }^{(b)}$ &,- 099 &,$- 286 * * *$ &,$- 235 * * *$ &,$- 225 * *$ & H5b: - \\
\hline Geschlecht (1 = männlich) &, $158 * *$ &, $306^{* * *}$ &, $244 * * *$ &, $211 * * *$ & \\
\hline Kind unter $15 \mathrm{im} \mathrm{HH}$ &, 105 &,- 023 &, 016 &, 024 & \\
\hline Kleinstadt ${ }^{(c)}$ &, $249 * *$ & ,108* & 069 &,$- 133^{*}$ & \\
\hline Großstadt $^{(\mathrm{d})}$ &, $242^{* * * *}$ &, $183^{* * *}$ & 057 &, 036 & \\
\hline Konstante & $1,604 * * *$ & $1,580 * * *$ & $1,598 * * *$ & $1,719 * * *$ & \\
\hline $\mathrm{N}$ & 1.736 & 5.114 & 5.984 & 2.385 & \\
\hline Wald chi ${ }^{2}(12)$ & $165,99 * * *$ & $453,92 * * *$ & $515,10 * * *$ & $282,50 * * *$ & \\
\hline \multicolumn{6}{|c|}{$\begin{array}{ll}\text { (a) Referenz: ledig } & * \text { signifikant auf } 5 \% \text {-Niveau } \\
\text { (b) Referenz: Hauptschulabschluss, kein Schulabschluss } & * * \text { signifikant auf } 1 \% \text {-Niveau } \\
\text { (c) 20.000-100.000 Einwohner (Referenz: unter 20.000 Einwohner) } & * * * \text { signifikant auf 0,1 \%-Niveau } \\
\text { (d) mehr als } 100.000 \text { Einwohner (Referenz: unter 20.000 Einwohner) } & \end{array}$} \\
\hline
\end{tabular}


Die Ergebnisse der Hurdle-Regressionen sind Tabelle $5 \mathrm{zu}$ entnehmen. ${ }^{22}$ Der obere Teil der Tabelle mit der Überschrift „Hurdle“ zeigt an, welche Faktoren sich positiv oder negativ auf das Überschreiten der Hürde Nichtrauchen $\left(\mathrm{n}_{\text {Zigaretten }}=0\right)$ versus Rauchen $\left(\mathrm{n}_{\text {Zigaretten }}\right.$ $>0$ ) auswirken. Die in diesem Tabellenabschnitt dargestellten Logits entsprechen folglich den Effektkoeffizienten auf den Rauchstatus (Hypothesen 1a bis 5a). Der untere Teil der Tabelle mit der Überschrift „Frequency“ zeigt die Ergebnisse der um Null gestutzten negativ binomialen Regression und damit den Effekt auf die Konsummenge (Hypothesen 1b bis 5b). Ein positives Vorzeichen bedeutet im ersten Tabellenabschnitt, dass die Wahrscheinlichkeit steigt überhaupt zu rauchen, im zweiten Tabellenabschnitt eine Steigerung der Konsummenge von Rauchern.

Die beiden Dummys für den Familienstand zeigen kein einheitliches Bild auf. Verheiratete Personen sind tendenziell seltener Raucher und wenn sie rauchen, neigen sie dazu, weniger Zigaretten zu rauchen als ledige Personen. Geschiedene oder verwitwete Personen sind tendenziell häufiger Raucher als ledige, jedoch unterscheidet sich die Konsummenge von geschiedenen bzw. verwitweten Rauchern nicht von derjenigen von ledigen Rauchern. Demzufolge kann Hypothese 1a tendenziell bestätigt werden und entspricht den bisherigen Befunden, während für Hypothese 1b kein empirischer Beleg gefunden wurde.

Der Erwerbsstatus wirkt sich über alle vier Datensätze hinweg signifikant auf den Rauchstatus aus, jedoch beeinflusst er nicht die Konsummenge von Rauchern. Erwerbslose sind zwar - analog zu Hypothese 2a und bisherigen Ergebnissen - häufiger Raucher als Erwerbstätige, erwerbslose Raucher weisen jedoch keine höheren Konsummengen als erwerbstätige Raucher auf. Demnach kann Hypothese 2b nicht bestätigt werden.

Für das Haushaltseinkommen prognostizieren Gewohnheits- und Suchttheorien einen positiven Effekt auf Rauchstatus und Konsummenge, wohingegen nach humankapitaltheoretischen Überlegungen ein negativer Effekt zu erwarten wäre. Der Effekt des Haushaltseinkommens auf den Rauchstatus weist zwar bei allen Datensätzen ein negatives Vorzeichen auf, ist allerdings nur bei der Analyse des BGS98 signifikant. Demnach sinkt die Wahrscheinlichkeit zu rauchen mit höherem Haushaltseinkommen der Personen. Gestützt wird dieses Ergebnis von den berichteten Analysen von Helmert et al. (2001) auf Grundlage des Mikrozensus 1995. Die humankapitaltheoretische Variante der Hypothese 3a kann folglich tendenziell bestätigt werden. Die Konsummenge von Rauchern variiert dagegen nicht mit der Höhe des Haushaltseinkommens (keine Bestätigung von Hypothese 3b).

Das Lebensalter wirkt sich sowohl auf den Rauchstatus als auch auf die Konsummenge aus (einzige Ausnahme ist der Effekt auf den Rauchstatus bei der Analyse mit dem EB95). Aus humankapitaltheoretischen Überlegungen wurde ein linearer, positiver Effekt auf die Rauchprävalenz abgeleitet. Aus Gründen einer korrekten Modellspezifikation wurde ein zusätzlicher quadratischer Term ergänzt. Der Alterseffekt auf die Konsummenge wird ebenfalls mit Hilfe von effect displays im Sinne von Fox (1987) visualisiert (siehe Abbildung 1).

22) Die in Tabelle 5 präsentierten Modelle wurden mit Stata 9.2 berechnet. Hurdle-Modelle sind nicht standardmäßig in Stata implementiert. Matthias Sinning vom Rheinisch-Westfälischen Institut für Wirtschaftsforschung (RWI) in Essen hat Stata-Befehle zur Berechnung sowohl von Hurdle-Poissonals auch von Hurdle-Negativ-Binomialmodellen (NB2-Modell nach Cameron / Trivedi 1986) geschrieben und uns zur Verfügung gestellt. Dafür bedanken wir uns herzlich. 
Abbildung 1: Anzahl der täglichen Zigarettenanzahl in Abhängigkeit von Alter und Geschlecht

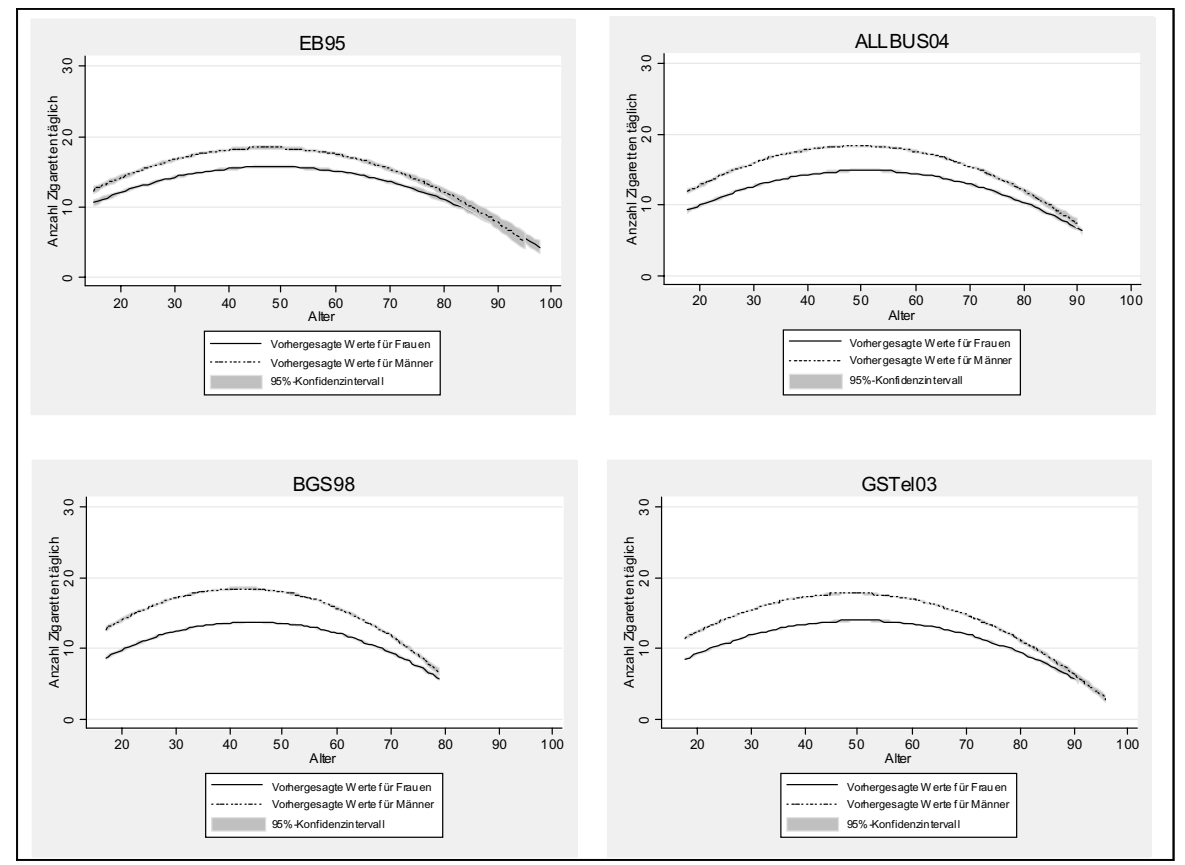

Es zeigt sich ein umgekehrt u-förmiger Effekt, der im Alter von ca. 50 Jahren seinen Scheitelpunkt erreicht. Das heißt, bis zu diesem Alter steigt der Konsum (sowohl die Wahrscheinlichkeit zu rauchen als auch die Konsummenge von Rauchern) mit zunehmendem Alter; ab dem Alter von ca. 50 Jahren sinkt der Konsum (die Hypothesen 4a und 4b werden also nur für das Alter bis 50 Jahre bestätigt). Der umgekehrt u-förmige Alterseffekt ist jedoch aufgrund der Querschnittsstruktur der Daten mit Vorsicht zu interpretieren. Der Effekt zeigt sich zwar sowohl hinsichtlich des Rauchstatus als auch bezüglich der Zigarettenmenge recht stabil; unklar bleibt allerdings, ob das Abfallen der Kurve durch eine tatsächliche Abnahme der Konsummenge ab einem gewissen Alter bedingt ist oder ob starke Raucher seltener ein hohes Alter erreichen und damit im Datensatz seltener vertreten sind (Survivaleffekt). ${ }^{23}$ Auch das Annähern der Rauchprävalenz von Frauen und Männern mit steigendem Alter (siehe Abbildung 1) könnte durch den Survivaleffekt bedingt sein. Um diesen Sachverhalt zu klären, bedarf es eines Paneldesigns mit genauer Dokumentation der wörtlich zu verstehenden „Panelmortalität“. Auch die bereits vorliegenden Befunde zu diesen Zusammenhängen können diese Lücke nicht schließen, da sie ebenfalls auf Querschnittsbetrachtungen beruhen. Insgesamt gehen diese Ergebnisse jedoch mit unseren Befunden einher (vgl. Abschnitt 2).

Der Effekt der Bildung kann weitgehend bestätigt werden (siehe Abbildung 2). Personen mit Abitur sind signifikant seltener Raucher als Personen mit Hauptschulabschluss oder keinem Schulabschluss und wenn sie rauchen, rauchen sie signifikant weniger Zigaretten als

23) Peto et al. (1996) schätzen eine um acht Jahre kürzere Lebensdauer von Gewohnheitsrauchern, Shaw et al. (2000) kommen zum Ergebnis, dass jede konsumierte Zigarette die Lebenszeit um elf Minuten reduziert. 
Personen mit niedriger Bildung. Für Personen mit mittlerer Bildung zeigt sich ein ähnlicher Effekt, jedoch ist dieser nicht vergleichbar stabil über alle vier Datensätze. Die schwache Erklärungskraft der Bildungsvariablen im Eurobarometer 1995 ist vermutlich bedingt durch die abweichende Messung des höchsten Bildungsabschlusses. Dennoch kann man von einer Bestätigung der Hypothesen 5a und 5b ausgehen.

Abbildung 2: Anzahl der täglichen Zigarettenanzahl in Abhängigkeit vom höchsten Schulabschluss

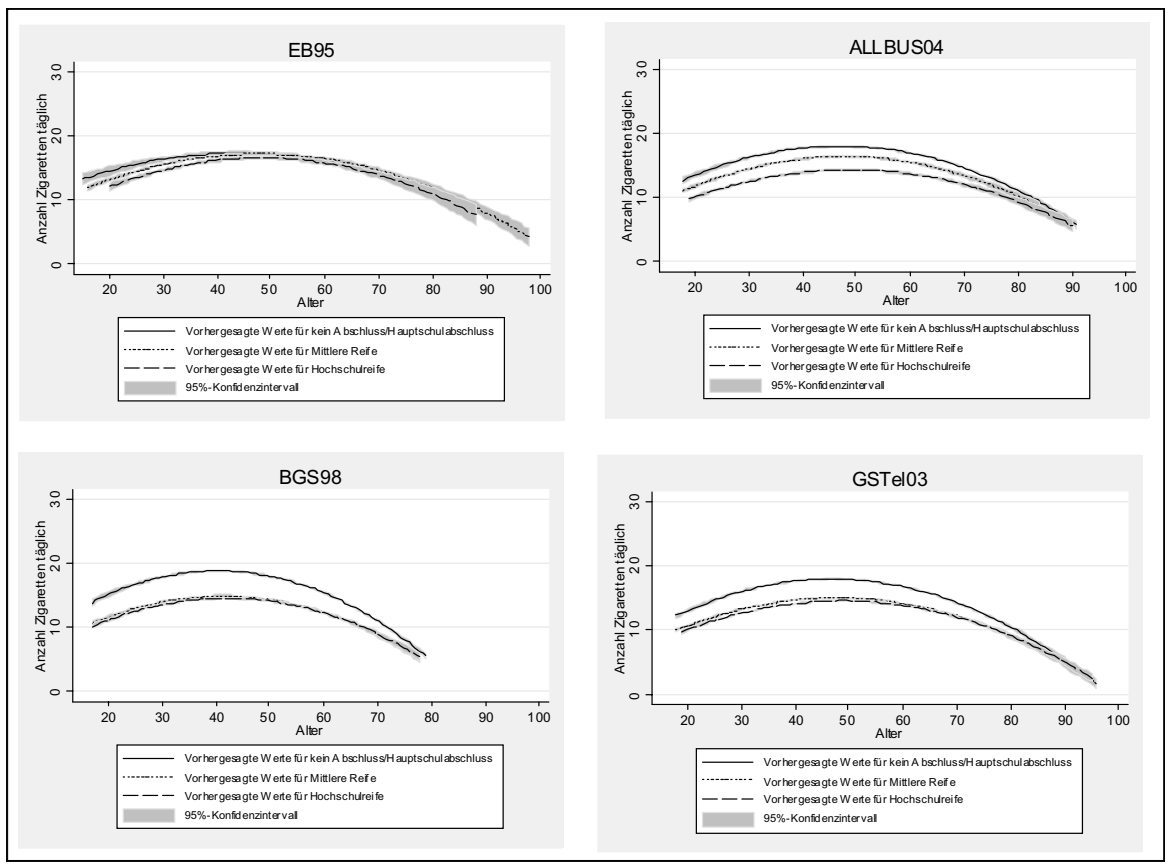

Die zum Schluss aufgeführten vier Variablen („Geschlecht“, „Kind unter 15 Jahren im Haushalt" und die beiden Dummys für die Wohnortgröße „Kleinstadt“ und „Großstadt“) wurden als Kontrollvariablen eingeführt. Das Geschlecht und die Wohnortgröße haben sich in bisherigen empirischen Studien als einflussreich gezeigt (vgl. Abschnitt 2), während die Anwesenheit zumindest eines Kindes im Haushalt aufgenommen wurde, um der Überlegung Rechnung zu tragen, dass der postulierte Alterseffekt möglicherweise ein versteckter Rücksichtseffekt sein könnte. Die Analysen zeigen, dass Männer signifikant häufiger Raucher sind als Frauen und die Konsummenge von Rauchern signifikant höher ist als die von Raucherinnen. Die Anwesenheit mindestens eines Kindes unter 15 Jahren im Haushalt wirkt sich weder auf den Rauchstatus noch auf die Konsummenge von Rauchern aus. Demnach kann die Rücksichtsvermutung nicht bestätigt werden und von der Existenz des Alterseffekts ausgegangen werden. Mit der Größe des Wohnortes steigen tendenziell die Wahrscheinlichkeit zu rauchen und die Anzahl der durchschnittlich gerauchten Zigaretten pro Tag. Der Effekt der Kleinstadt auf die Konsummenge ist über die Datensätze hinweg widersprüchlich. Während die Analysen des EB95 und der BGS98 von einem signifikant positiven Effekt auf die Konsummenge ausgehen, beschreibt der ALLBUS04 einen negativen Effekt. 


\section{Resümee}

Die hier präsentierten Analysen haben zum einen gezeigt, dass aus den beiden behandelten Theorieansätzen des Rational-Choice-Paradigmas brauchbare Hypothesen zum Rauchverhalten abgeleitet werden können und diese zum anderen auch empirische Relevanz besitzen.

Im Einzelnen postulieren die herangezogenen Gewohnheits- und Suchtmodelle konsumförderliche Effekte von so genannten Lebenszyklusschocks, die mit Hilfe der Variablen ,geschieden / verwitwet“ und „derzeit arbeitslos“ untersucht wurden. Diese Effekte bestätigen sich tendenziell für den Rauchstatus, jedoch nicht für die Zigarettenmenge. Der Anstieg der Zigarettenmenge von Rauchern nach solchen Schocks lässt sich mit Querschnittsdatensätzen nicht optimal untersuchen. Insofern wird man Gewohnheits- und Suchtmodellen mit dieser Vorgehensweise nicht vollkommen gerecht, da der dynamische Prozess vernachlässigt wird. Neben Gewohnheits- und Suchtmodellen sprechen diese Ergebnisse auch für alternative theoretische Erklärungen wie etwa dem Belastungs-Bewältigungs-Paradigma, auf das nicht näher eingegangen wurde.

Theoretische Folgerungen aus dem Humankapitalansatz und dem Nachfragemodell nach health capital beschreiben einen Anstieg der Rauchwahrscheinlichkeit und Menge mit geringer Bildung, steigendem Alter und geringem Einkommen. Der Effekt hoher Bildung, der den Kern der Theorie darstellt, bleibt über alle Datensätze hinweg stabil. Der Einkommenseffekt weist ebenfalls in die erwartete Richtung, ist jedoch nicht so robust. Für junge Altersgruppen bestätigt sich, dass die Wahrscheinlichkeit zu rauchen und die Konsummenge mit steigendem Alter zunehmen. Das Abfallen der Kurve ab einem gewissen Alter müsste zusätzlich im Längsschnittdesign untersucht werden. Gleichfalls könnten diese Ergebnisse im Rahmen des Health-Belief-Modells interpretiert werden, wenngleich damit einige methodische Probleme verbunden sind (siehe Fußnote 14).

Diesen Befunden zufolge erscheint es demnach sinnvoll, Rational-Choice-Ansätze bei der Erklärung von Gewohnheits- und Suchtverhalten zu berücksichtigen. Für weitere Forschungsbemühungen zum Thema Rauchen schlagen wir jedoch Verbesserungsmöglichkeiten in zweifacher Hinsicht vor: Zum einen sollten die Theorieansätze dahingehend weiterentwickelt werden, dass die Differenzierung zwischen Rauchstatus und Zigarettenmenge ermöglicht wird, wie dies im Rahmen der Gewohnheits- und Suchtmodelle schon ansatzweise geschehen ist. Es bleibt theoretisch ungeklärt, weshalb sich Faktoren wie etwa Familienstand, Erwerbsstatus und Einkommen auf den Rauchstatus, jedoch nicht auf die Zigarettenmenge auswirken. Zum anderen sollten Bevölkerungsumfragen zum Thema Rauchen möglichst umfassend die für einen eingehenden Theorientest notwendigen Variablen erheben. So enthält keiner der verfügbaren Datensätze detaillierte Angaben zu Raucherkarrieren und in nur zwei der vier verwendeten wurden Faktoren zum Rauchverhalten des sozialen Umfeldes erhoben (und selbst das in keinster Weise befriedigend). Dies erscheint insofern verwunderlich, als diese Variablen sowohl in Form von Netzwerkansätzen theoretisch eingebunden sind als auch sich in bisherigen Analysen empirisch bewährt haben (für Jugendliche siehe bspw. Kraus et al. 2004). Der Zigarettenpreis, der sich in Folge von Schmuggel und niedrigeren Zigarettenpreisen in Nachbarländern nicht so einheitlich gestaltet wie meist angenommen, wurde in keinem der Datensätze erhoben. Auch diesbezüglich scheint die Theoriearbeit keine Auswirkungen auf die empirische Umsetzung von Bevölkerungsumfragen zu haben, obwohl US-amerikanische Studien die empirische Evidenz des Zusammenhangs aufzeigen (siehe etwa Becker et al. 1991). Sowohl die Theoriearbeit als auch die empirische Umsetzung von Bevölkerungsumfragen weisen folglich noch Verbesserungspotenzial für ein umfassenderes Verständnis des Rauchverhaltens auf. 


\section{Literatur}

Bauer, Thomas / Silja Göhlmann / Mathias Sinning (2006): Gender Differences in Smoking Behavior, RWI Discussion Paper Nr. 44, Essen.

Becker, Gary S. (1992): Habits, Addiction, and Traditions, in: Kyklos 45, S. 327-346.

Becker, Gary S. (1993 [1964]): Human Capital. A Theoretical and Empirical Analysis with Special Reference to Education, 3. Aufl., Chicago.

Becker, Gary S. (1996): Accounting for Tastes, Cambridge - London.

Becker, Gary S. / Kevin M. Murphy (1988): A Theory of Rational Addiction, in: Journal of Political Economy 96, S. 675-700.

Becker, Gary S. / Michael Grossman / Kevin M. Murphy (1991): Rational Addiction and the Effect of Price on Consumption, in: American Economic Review 81, S. 237-241.

Becker, Gary S. / Michael Grossman / Kevin M. Murphy (1994): An Empirical Analysis of Cigarette Addiction, in: The American Economic Review 84, S. 396-418.

Becker, Marshall H. (Hrsg.) (1974): The Health Belief Model and Personal Health Behavior. Health Education Monographs 2, Thorofare.

Bellach, Bärbel-Maria / Hildtraud Knopf / Wolfgang Thefeld (1998): Der Bundes-Gesundheitssurvey 1997 / 1998, in: Gesundheitswesen 60, Sonderheft 2, S. 59-68.

Berger, Roger (2003): Gewohnheit, Sucht und Tradition, Leipzig.

Best, Henning (2007): Die Messung von Nutzen und subjektiven Wahrscheinlichkeiten. Ein Vorschlag zur Operationalisierung der Rational Choice Theorie, in: Methoden - Daten - Analysen 1, S. 183-212.

Boudon, Raymond (1998): Beyond Rational Choice Theory, in: Annual Review of Sociology 29, S. 1-21.

Braun, Norman (2002): Rationalität und Drogenproblematik, München.

Braun, Norman / Paolo Vanini (2003): On Habits and Addictions, in: Journal of Institutional and Theoretical Economics 159, S. 603-626.

Brenner, Hermann / Sebastian Schneeweiss / Manfred Wildner / Uwe Siebert / Eva Grill (2002): Handbuch Epidemiologie, PH EBOOX Nr. 2001-05, München, abgerufen am 26.02.2007 unter http:// www.umit.at/upload/msc2_modul_gx.pdf.

Bundeszentrale für gesundheitliche Aufklärung (BZgA) (2006): Förderung des Nichtrauchens. Eine Wiederholungsbefragung der Bundeszentrale für gesundheitliche Aufklärung, Köln, abgerufen am 27.02.2007 unter http://www.bzga.de/?uid=b3a800ccee97528c4646c29b393c8428\&id=Seite1417.

Burgess, Robert L. / Ronald L. Akers (1966): A Differential Association - Reinforcement Theory of Criminal Behavior, in: Social Problems 14, S. 128-147.

Cameron, Adrian C. / Pravin K. Trivedi (1998): Regression Analysis of Count Data, Cambridge.

Chaloupka, Frank (1991): Rational Addictive Behavior and Cigarette Smoking, in: Journal of Political Economy 99, S. 722-742.

Dicken, Charles (1978): Sex Roles, Smoking, and Smoking Cessation, in: Journal of Health and Social Behavior 19, S. 324-334.

Dockner, Engelbert / Gustav Feichtinger (1993): Cyclical Consumption Patterns and Rational Addiction, in: American Economic Review 83, S. 257-263.

Eifler, Stefanie (1997): „Gleich und gleich gesellt sich gern!“ - Eine empirische Analyse der Einflüsse von Self-Control und differentiellen Assoziationen auf den Genuß von Tabakwaren, in: Soziale Probleme 8, S. 199-217.

Eifler, Stefanie (1998): Machen Gelegenheiten Raucher? Theoretische und empirische Analysen der Beziehungen zwischen Self-Control, Ressourcen und dem Genuß von Tabakwaren, in: Soziale Probleme 9, S. 90-116.

Elkind, Andrea K. (1985): The Social Definition of Women Smoking Behaviour, in: Social Science \& Medicine 20, S. 1269-1278. 
Elster, Jon (1993): Some Unresolved Problems in the Theory of Rational Behavior, in: Acta Sociologica 36, S. 179-190.

Elster, Jon / Ole-Jørgen, Skog (1999): Introduction, in: Dies. (Hrsg.), Getting Hooked. Rationality and Addiction, Cambridge, S. 1-29.

Esser, Hartmut (1990): „Habits“, „Frames“ und „Rational Choice“. Die Reichweite von Theorien der rationalen Wahl (am Beispiel der Erklärung des Befragtenverhaltens), in: Zeitschrift für Soziologie 19, S. 231-247.

Esser, Hartmut (2001): Soziologie. Spezielle Grundlagen Band 6: Sinn und Kultur, Frankfurt / Main New York.

Esser, Hartmut (2002): In guten wie in schlechten Tagen? Das Framing der Ehe und das Risiko zur Scheidung, in: Kölner Zeitschrift für Soziologie und Sozialpsychologie 54, S. 27-63.

European Commission (1995): Eurobarometer 43.0, Cross-boarder Purchases, Smoking Habits and Cancer Risks, March-April 1995.

Ferrence, Roberta G. (1988): Sex Differences in Cigarette Smoking in Canada, 1900-1978: A Reconstructed Cohort Study, in: Canadian Journal of Public Health 79, S. 160-165.

Fox, John (1987): Effect Displays for Generalized Linear Models, in: Sociological Methodology 17, S. 347-361.

Frey, Bruno S. / Reiner Eichenberger (1989): Should Social Scientists Care About Choice Anomalies?, in: Rationality and Society 1, S. 101-122.

Gabler, Siegfried / Sabine Häder (1998): Ein neues Stichprobendesign für telefonische Umfragen in Deutschland, in: Siegfried Gabler / Sabine Häder / Jürgen H.-P. Hoffmeyer-Zlotnik (Hrsg.), Telefonstichproben in Deutschland, Wiesbaden, S. 101-119.

Gardner, Eliot L. / James David (1999): The Neurobiology of Chemical Addiction, in: Jon Elster / OleJørgen Skog (Hrsg.), Getting Hooked. Rationality and Addiction, Cambridge, S. 93-136.

Gjelsvik, Olav (1999): Addiction, Weakness of the Will, and Relapse, in: Jon Elster / Ole-Jørgen Skog (Hrsg.), Getting Hooked. Rationality and Addiction, Cambridge, S. 47-64.

Godeau, Emmanuelle / Giora Rahav / Anna Hublet (2004): Tobacco Smoking, in: Candace Currie / Chris Roberts / Antony Morgan / Rebecca Smith / Wolfgang Settertobulte / Oddrun Samdal / Vivian Barnekow Rasmussen (Hrsg.), Young People's Health in Context. Health Policy for Children and Adolescents, Nr. 4, Kopenhagen, S. 63-72.

Gottfredson, Michael R. / Travis Hirschi (1990): A General Theory of Crime, Stanford.

Green, Donald P. / Ian Shapiro (1994): Pathologies of Rational Choice Theory. A Critique of Applications in Political Science, New Haven u.a.

Grossman, Michael (1972): On the Concept of Health Capital and the Demand for Health, in: Journal of Political Economy 80, S. 223-255.

Grossman, Michael (1995): The Economic Approach to Addictive Behavior, in: Michael Tommasi / Katheryn Ierulli (Hrsg.), The New Economics of Human Behavior, Cambridge, S. 151-171.

Haarmann, Alexander / Evi Scholz / Martina Wasmer / Michael Blohm / Janet Harkness (2006): Konzeption und Durchführung der „Allgemeinen Bevölkerungsumfrage der Sozialwissenschaften“ (ALLBUS) 2004, Mannheim, abgerufen am 16.06.2006 unter http://www.gesis.org/Dauerbeobachtung/ Allbus/documents/pdfs/mb2004.pdf.

Helmert, Uwe (1999): Einkommen und Rauchverhalten in der Bundesrepublik Deutschland - eine Sekundäranalyse der Daten des Mikrozensus 1995, in: Gesundheitswesen 61, S. 31-37.

Helmert, Uwe / Dieter Borgers (1998): Rauchen und Beruf - Eine Analyse von 100000 Befragten des Mikrozensus 1995, in: Bundesgesundheitsblatt 41, S. 102-107.

Helmert, Uwe / Dieter Borgers / Karin Baumann (2001): Soziale Determinanten des Rauchverhaltens in Deutschland: Ergebnisse des Mikrozensus 1995, in: Sozial- und Präventivmedizin 46, S. 172-181.

Helmert, Uwe / Ulrike Maschewsky-Schneider (1998): Zur Prävalenz des Tabakrauchens bei Arbeitslosen und Armen, in: Dieter Henkel (Hrsg.), Sucht und Armut. Alkohol, Tabak, illegale Drogen, Opladen, S. 153-165. 
Herrnstein, Richard J. / Drazen Prelec (1992): A Theory of Addiction, in: George Loewenstein / Jon Elster (Hrsg.), Choice Over Time, New York, S. 331-360.

John, Ulrich / Monika Hanke (2001): Tabakrauch-attributable Mortalität in den deutschen Bundesländern, in: Das Gesundheitswesen 63, S. 363-369.

Janz, Nancy / Victoria L. Champion / Victor J. Strecher (2002): The Health Belief Model, in: Karen Glanz / Barbara K. Rimer / Frances Marcus Lewis (Hrsg.), Health Behavior and Health Education, 3. Aufl., San Francisco, S. 45-66.

Jungbauer-Gans, Monika / Christiane Gross (2006): Erklärungsansätze sozial differenzierter Gesundheitschancen, in: Matthias Richter / Klaus Hurrelmann (Hrsg.), Gesundheitliche Ungleichheit. Grundlagen, Probleme, Perspektiven, Wiesbaden, S. 73-89.

Junge, Burckhard / Matthias Nagel (1999): Das Rauchverhalten in Deutschland, Gesundheitswesen 61 (Supplement), S. 121-125.

Kohler, Martin (2005): Stichprobenkonzeption und Stichprobenrealisierung, in: Robert Koch-Institut (Hrsg.), Erster telefonischer Gesundheitssurvey des Robert Koch-Instituts - Methodische Berichte, Berlin, S. 31-57.

Kohler, Martin / Thomas Ziese (2004): Telefonischer Gesundheitssurvey des Robert Koch-Instituts zu chronischen Krankheiten und ihren Bedingungen, Berlin.

Kraus, Ludwig / Rita Augustin (2001): Repräsentativerhebung zum Gebrauch psychoaktiver Substanzen bei Erwachsenen in Deutschland 2000, in: Sucht 47, Sonderheft 1, S. 1-86.

Kraus, Ludwig / Kathrin Heppekausen / Andrea Barrera / Boris Orth (2004): Die Europäische Schülerstudie zu Alkohol und anderen Drogen (ESPAD): Befragung von Schülerinnen und Schülern der 9. und 10. Klasse in Bayern, Berlin, Brandenburg, Hessen, Mecklenburg-Vorpommern und Thüringen, IFTBerichte Band 141, München.

Lampert, Thomas (2005): Tabakkonsum und soziale Ungleichheit. Gesundheitspolitische Konsequenzen des stärkeren Tabakkonsums in sozial benachteiligten Bevölkerungsgruppen, in: Konturen, S. 15-17.

Lampert, Thomas / Burger, Martin (2004): Rauchgewohnheiten in Deutschland - Ergebnisse des telefonischen Bundes-Gesundheitssurveys 2003, in: Gesundheitswesen 66, S. 511-517.

Lampert, Thomas / Martin Burger (2005): Verbreitung und Strukturen des Tabakkonsums in Deutschland, in: Bundesgesundheitsblatt - Gesundheitsforschung - Gesundheitsschutz 48, S. 1231-1241.

Lampert, Thomas / Michael Thamm (2004): Soziale Ungleichheit des Tabakkonsums in Deutschland, in: Bundesgesundheitsblatt - Gesundheitsforschung - Gesundheitsschutz 47, S. 1033-1042.

Long, J. Scott (1997): Regression Models for Categorial and Limited Dependent Variables, in: Advanced Quantitative Techniques in the Social Sciences 7, Thousand Oaks.

Long, J. Scott / Jeremy Freese (2006): Regression Models for Categorial Dependent Variables Using Stata. 2. Aufl., College Station.

Molarius, Anu / Richard W. Parsons / Annette J. Dobson / Alun Evans / Stephen P. Fortmann / Konrad Jamrozik / Kari Kuulasmaa / Vladislav Moltchanov / Susana Sans / Jakko Tuomilehto / Pekka Puska (2001): Trends in Cigarette Smoking in 36 Populations from the Early 1980s to the Mid-1990s: Findings from the WHO MONICA Project, in: American Journal of Public Health 91, S. 206-212.

Orphanides, Athanasios / David Zervos (1995): Rational Addiction with Learning and Regret, in: Journal of Political Economy 103, S. 739-758.

Orphanides, Athanasios / David Zervos (1998): Myopia and Addictive Behaviour, in: The Economic Journal 108, S. 75-91.

Peto, Richard / Alan D. Lopez / Jilian Boreham / Michael Thun (1994): Mortality from Smoking in Developed Countries 1950-2000: Indirect Estimates from National Vital Statistics, Oxford.

Peto, Richard / Alan D. Lopez / Jilian Boreham / Michael Thun / Clarke Heath Jr. / Richard Doll (1996): Mortality from Smoking Worldwide, in: British Medical Bulletin 52, S. 12-21.

Potthoff, Peter / Ernst Schroeder / Ursula Reis / Angelika Klamert (1999): Ablauf und Ergebnisse der Feldarbeit beim Bundes-Gesundheitssurvey, in: Gesundheitswesen 61, Sonderheft 2, S. 62-67.

Public Use File GSTe103, telefonischer Gesundheitssurvey (2003), Robert Koch-Institut, Berlin. 
Reif, Karlheinz / Eric Marlier (1996): Eurobarometer 43.0: Drug Abuse and AIDS, October-November 1995.

Robert-Koch-Institut (2000): Public Use File Bundes-Gesundheits-Survey 1998, Berlin.

Shaw, Mary / Richard Mitchell / Danny Dorling (2000): Time for a Smoke? One Cigarette Reduces Your Life by 11 Minutes, in: British Medical Journal 320, S. 53.

Skog, Ole-Jørgen (1999): Rationality, Irrationality, and Addiction - Notes on Becker's and Murphy's Theory of Addiction, in: Jon Elster / Ole-Jørgen Skog (Hrsg.), Getting Hooked. Rationality and Addiction, Cambridge, S. 93-136.

Thefeld, Wolfgang / Heribert Stolzenberg / Bärbel-Maria Bellach (1999): Bundes-Gesundheitssurvey: Response, Zusammensetzung der Teilnehmer und Non-Responder-Analyse, in: Gesundheitswesen 61, Sonderheft 2, S. 57-61.

Thierfelder, Wulf / Charlotte Seher / Wolfgang Thefeld (1998): Der Bundes-Gesundheitssurvey 1997 / 1998 - Untersuchungsteil, in: Gesundheitswesen 60, Sonderheft 2, S. 69-76.

Völzke, Henry / Hanne Neuhauser / Susanne Moebus / Jens Baumert / Klaus Berger / Andreas Stang / Ute Ellert / André Werner / Angela Döring (2006): Rauchen: Regionale Unterschiede in Deutschland, in: Deutsches Ärzteblatt 103: S. 2784-2790.

Waldron, Ingrid (1991): Patterns and Causes of Gender Differences in Smoking, in: Social Science \& Medicine 32, S. 989-1005.

Warschburger, Sabine (2002): Rauchen und Alterssicherung, Frankfurt / Main.

Winkelmann, Rainer (2003): Econometric Analysis of Count Data, 4. Aufl., Berlin u.a.

Winkler, Gertrud / Birgit Filipiak / Hans-Werner Hense / Bernhard Schwertner (1999): Externe Qualitätskontrolle im Bundes-Gesundheitssurvey: Konzept und Site-Visits, in: Gesundheitswesen 61, Sonderheft 2, S. 68-71.

Zentralarchiv für Empirische Sozialforschung: Allgemeine Bevölkerungsumfrage der Sozialwissenschaften ALLBUS 2004, Köln.

Ziese, Thomas (2005): Der telefonische Gesundheitssurvey - Bausteine zum Gesundheitsmonitoringsystem für Deutschland, in: Robert Koch-Institut (Hrsg.), Erster telefonischer Gesundheitssurvey des Robert Koch-Instituts - Methodische Berichte, Berlin, S. 5-8.

Christiane Gross

Christian-Albrechts-Universität zu Kiel Institut für Sozialwissenschaften

Westring 400

24098 Kiel

cgross@soziologie.uni-kiel.de

Jochen Groß

Ludwig-Maximilians-Universität München Institut für Soziologie

Konradstraße 6

80801 München

jochen.gross@ soziologie.uni-muenchen.de 